TM-1653

\title{
Conceptual Design for the SSC High Energy Booster*
}

T. H. Nicol, F. A. Harfoush, M. A. Harrison, J. S. Kerby, K. P. Koepke, P. M. Mantsch, T. J. Peterson, and A. W. Riddiford

Fermi National Accelerator Laboratory P.O. Box 500

Batavia, Illinois 60510

March 1990

* Presented by T. H. Nicol at the 1990 International Industrial Symposium on the Super Collider (IISSC), Miami

Beach, Florida, March 14-16, 1990. 


\title{
CONCEPTUAL DESIGN FOR THE SSC HIGH ENERGY BOOSTER
}

\author{
T.H. Nicol, F.A. Harfoush, M.A. Harrison, J.S. Kerby \\ K. P. Koepke, P.M. Mantsch, T.J. Peterson, and \\ A.W. Riddiford \\ Fermilab National Accelerator Laboratory \\ Box 500 \\ Batavia, IL 60510
}

\begin{abstract}
A tremendous amount of work has been done over the course of the past several years on the design of dipole magnets for the SSC main collider. Although they dominate the total magnet cost for the project and thus deserve the tremendous research and development effort they have received, the main ring magnets represent only part of the magnet requirements for the SSC. This paper presents the work to date on the design of dipole magnets for the SSC high energy booster (HEB). A complete discussion of the design is beyond the scope of this work. ${ }^{1}$ Rather it serves as an overview of the main aspects of the complete design.
\end{abstract}

\section{INTRODUCTION}

The high energy booster serves as the injector for the main collider ring as well as a source for test beams. The dipoles for the HEB are required to produce a peak operating field of 6.26 T which corresponds to a maximum energy of $2 \mathrm{Tev}$. The injection energy of $200 \mathrm{Gev}$ defines the minimum field level of $0.626 \mathrm{~T}$. The magnet design is consistent with the overall goals of the accelerator operation which require continuous ramping with a two minute cycle. The relationship of the HEB to each of the other components of the complete accelerator are shown in Figure 1.

The design requirements for the HEB magnets differ from those of the main collider in several respects. The good field aperture requirement gives a bore that is $40 \%$ larger; $70 \mathrm{~mm}$ compared to $50 \mathrm{~mm}$ for the collider dipole and the magnetic field strength is slightly less; $6.26 \mathrm{~T}$ as compared to $6.6 \mathrm{~T}$ for the collider. The HEB must also be capable of bipolar operation and continuous ramping and must be stable up to $10 \%$ above its nominal operating field. The design of the HEB dipole includes a cable specification which ensures adequate operating margin, coil and iron geometries to provide field shape, mechanical structures to contain coil stresses both from fabrication preload and Lorentz forces, and a complete cryostat system. This paper will address each of these topics in some detail with special emphasis on some of the more interesting aspects of the design. Table 1 lists as many of the design parameters as we were able to deduce from a preliminary specification of the HEB magnet system or to conclude as a result of our design work. Figure 2 illustrates a two dimensional cross section through the complete magnet assembly developed during the course of this study. 


$\begin{array}{ll}\text { Number of dipoles } & 864 \\ \text { Overall dipole slot length } & 8.25 \mathrm{~m} \\ \text { Magnetic length } & 7.75 \mathrm{~m} \\ \text { Coil inner diameter } & 70 \mathrm{~mm} \\ \text { Mass of conductor } & 363 \mathrm{~kg} \\ \text { Cold Mass } & 6708 \mathrm{~kg} \\ \text { Central Field } & 6.26 \mathrm{~T} \\ \text { Current } & 5582 \mathrm{~A} \\ \text { Inductance } & 80 \mathrm{mH} \\ \text { Stored energy } & 1.25 \mathrm{MJ} \\ \text { Beam tube inner diameter } & 56 \mathrm{~mm} \\ \text { Inner layer number of turns } & 72 \\ \text { Outer layer number of turns } & 60 \\ \text { Iron Yoke inner diameter } & 20 \mathrm{~cm} \\ \text { Iron Yoke outer diameter } & 42 \mathrm{~cm} \\ \text { Cryostat outer diameter } & 72 \mathrm{~cm} \\ \text { Magnet mass } & 7973 \mathrm{~kg}\end{array}$

\section{COIL CROSS SECTION}

The most significant change to the coil cross section is the introduction of an offset coil design which improves the profile of the field error, $\mathrm{dB} / \mathrm{B}$. The idea has been studied by $\mathrm{T}$. Collins ${ }^{2}$, and more recently by Ishibashi ${ }^{3}$. They studied the simplified problem of one constant current density area per group of conductors, neglecting the insulation wrapped around each conductor.

The conductor is assumed to have the same critical current density as the conductor used in the collider dipole $\left(2750 \mathrm{~A} / \mathrm{mm}^{2}\right.$ at $5 \mathrm{~T}$ and $4.2 \mathrm{~K}$ ). The primary objective in selecting the conductor for this magnet was to ensure that the magnet have a performance margin of $10 \%$ over the nominal operating field. A copper-to-superconductor ratio of 1.5:1 was selected to ensure coil stability and to facilitate quench protection.

Figure 3 shows a typical cross-section through the coil assembly. A wedge is not needed in the outer layer for the given coil inner diameter. The keystone angle is large enough to keep the conductors roughly radial. However, the inner layer of conductors needs at least one wedge to keep conductors radial. It has been found that two wedges permit solutions with smaller harmonic coefficients. Furthermore, each of the wedges

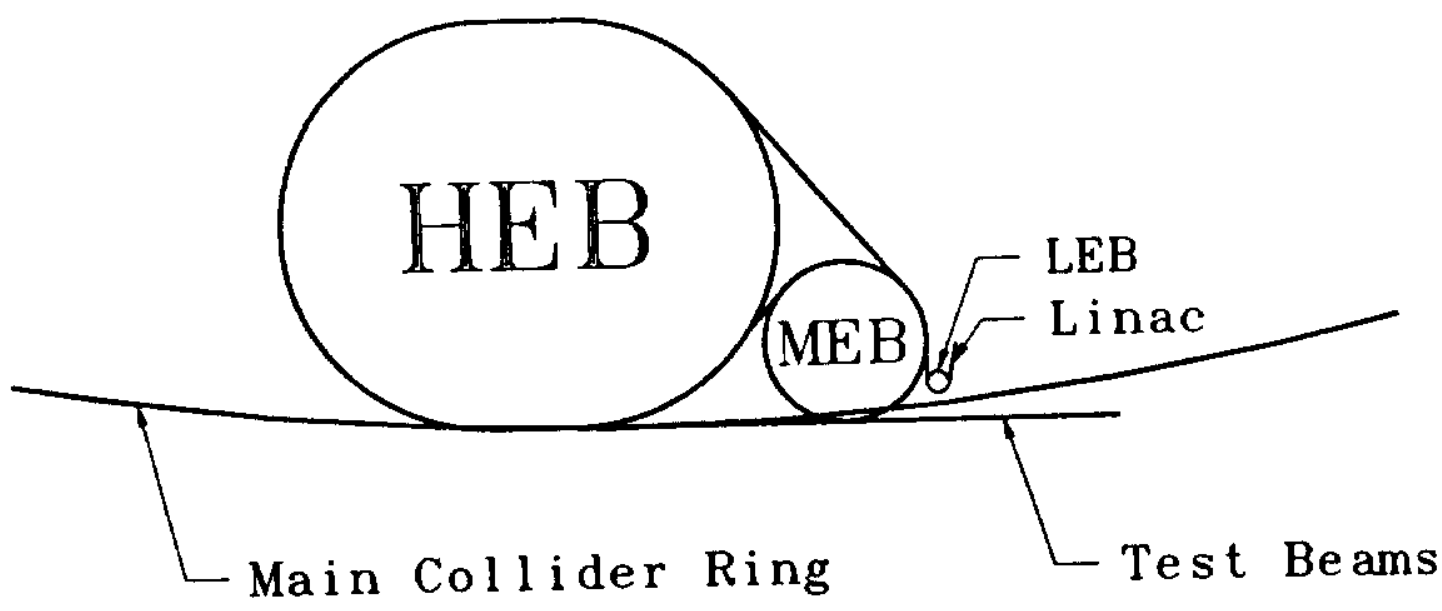

Figure 1. SSC Accelerator Components 


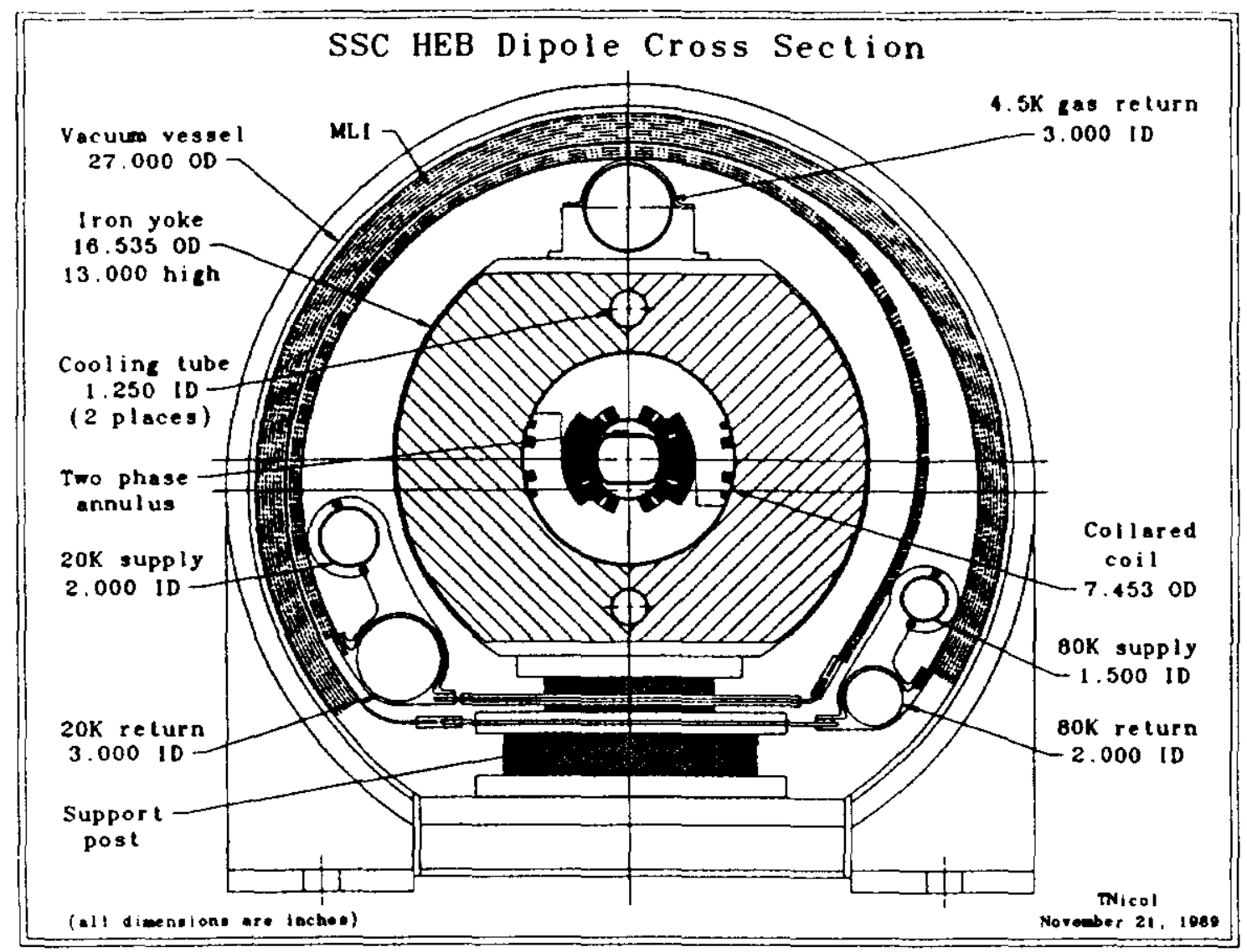

Figure 2. Complete HEB Two Dimensional Cross Section

allows a solution to be found which zeros out one of the coefficients. The method discussed here uses two wedges and zeros out the sextupole and decapole harmonic coefficients. The magnet performance is summarized in Table 2.

Table 2. HEB Dipole Magnet Performance

Imax (amps)

6200

$B \max (\mathrm{T})$

7.521

B (@Imax) central field (T)

7.021

Bmax/B central

$7.14 \%$

I operating current (amps)

5582

B (QI operating) central field (T)

6.26

Transfer function (Tesla/kA)

1.132

Number of turns

132

Inner iron radius (inches)

3.789

Coil offset (inches)

0.163

$\mathrm{dB} / \mathrm{Bmax}(\mathrm{x} 10000)$

1.006

Radius at $\mathrm{dB} / \mathrm{Bmax}$ (inches)

1.05

Harmonic coefficients at 1 inch in units of $10^{-4}$ :

$\begin{array}{ll}2 \text { pole: } & 10000 \\ 6 & 0.0 \\ 10 & 0.0 \\ 14 & 0.42 \\ 18 & 1.62 \\ 22 & 0.70\end{array}$

$\begin{array}{ll}26 \text { pole: } & -2.18 \\ 30 & 0.42 \\ 34 & -0.02 \\ 38 & 0.01 \\ 42 & 0.03\end{array}$




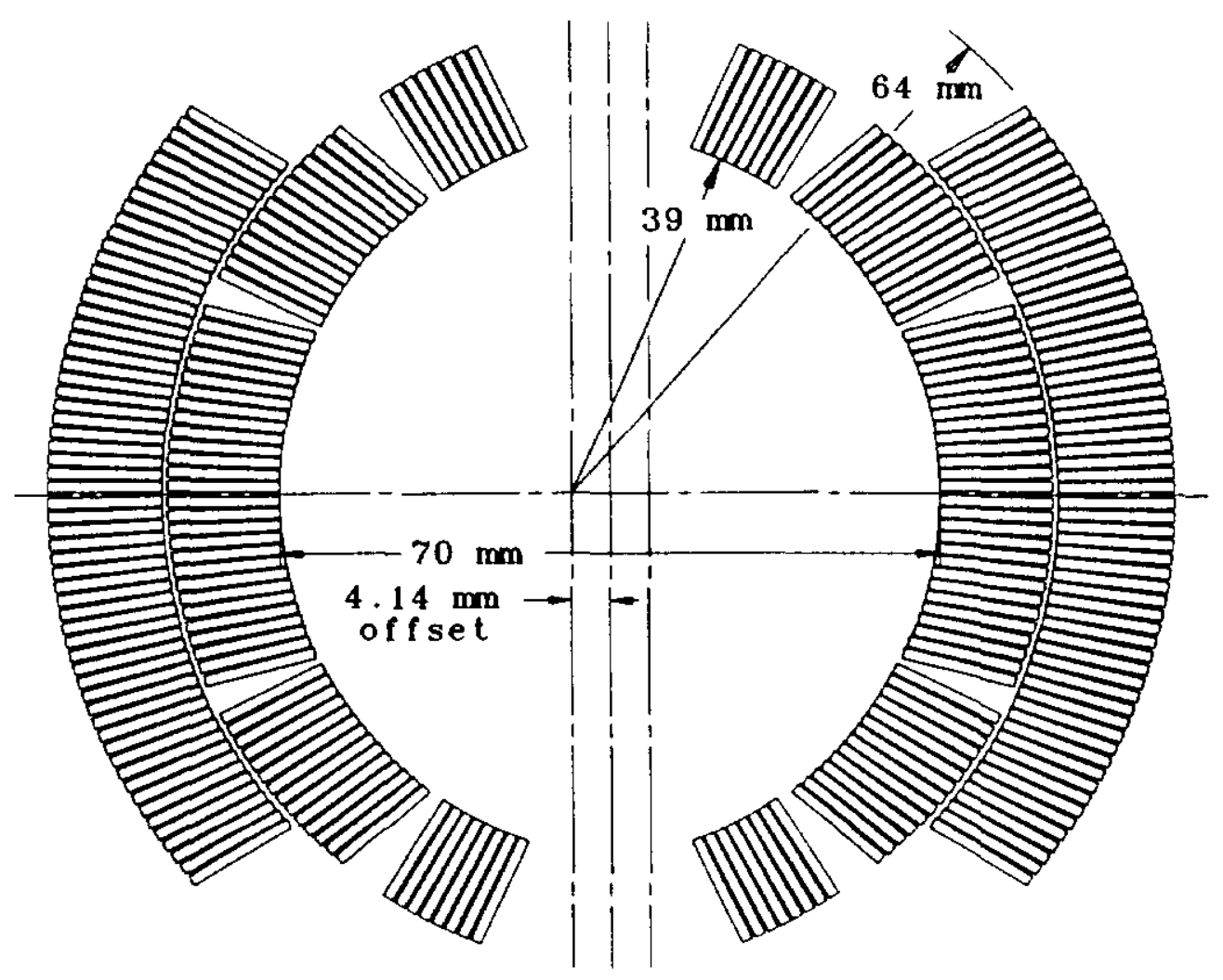

Figure 3. HEB Offset Coil Geometry

\section{YOKE MAGNETIC DESIGN}

The iron yoke, in addition to holding the coils rigidly in place acts to give a substantial field enhancement. The iron contribution to the field can be analytically computed for a finite or infinite permeability. The problem becomes analytically intractable when the permeability itself becomes a function of field, resulting in a nonlinear problem. Numerical methods, such as POISSON, have been widely used to solve such problems. Our main concern here in designing the yoke is to achieve a level of saturation sextupole less than 2 units at 1 inch and an amplification factor of less than $2 \%$. A convenient unit of measurement of the multipole coefficients is in units of $10^{-4} \mathrm{~B}_{0}$, that is, one part in 10,000 of the main dipole field. Since this magnet is to have a bipolar operation, an important question is the effect of remnant field on the field quality. We shall address all these questions and explain the steps that lead to our suggested yoke design.

First we looked at the effect of varying the iron inner radius on the sextupole. It has been observed from numerical simulations that for a given inner radius the sextupole variation versus dipole field can have one of two shapes. The first and most common shape is where the sextupole magnitude increases to a maximum value and then decreases. This behavior is attributed to the saturation in the immediate vicinity of the coil causing the increase in sextupole. Saturation on either side of the coil will cause the sextupole magnitude to decrease. It is important to note that such a behavior is very dependent on the inner radius value. For a relatively large inner radius, the notion of immediate vicinity becomes less significant. This results in a reduction in the peak value until a point is reached where any increase in inner radius will lead to the second shape; that of a monotonically decreasing sextupole versus dipole field. 
An increase in inner radjus drives the sextupole more negative and a decrease drives it more positive. This implies that the negative contribution is due to the midplane saturation and the positive contribution to the pole saturation nearest to the coil. We have found that for an inner radius of 4 inches, the sextupole value at both low and high fields is negative.

As the inner radius increases the curve becomes a monotonically decreasing function. Based on the two types of behavior just discussed we then have two options in our yoke design. Either select a radius such that the sextupole value over a given field range does not exceed a limit value or have a monotonically decreasing value that will not reach a critical value at a desired high field point. Increasing the iron inner radius will decrease the iron saturation and therefore result in a better amplification factor. The opposite is true if we decrease the inner radius. An inner radius in the range of 3.5 to 4 inches will limit the amplification factor to less than $2 \%$ at $6.6 \mathrm{~T}$ fields. The effect of the outer radius on sextupole is less predictable.

By reducing the thickness of the iron we will significantly increase the sextupole component. The effect of the outer radius on the dipole field quality is measured by the amplification factor. By increasing the thickness of the iron a smaller amplification factor (stronger dipole field) is obtained up to a point beyond which any increase in radius will have little effect on the field and the outer radius acts as if it were infinite. The opposite is true if we reduce the thickness of the iron.

Another important parameter in the yoke design are the fringe fields. Looking at the midplane cross section along the horizontal axis it is possible to plot the field value in air in the immediate vicinity of the iron. For an outer radius of 8.66 inches the fringe field is less than $1 \mathrm{kG}$. We have chosen this value as our minimum outer yoke radius.

To summarize the result of our findings the inner radius of the iron should be selected in the range of 3.5 to 4 inches and the outer radius should have a minimum radius of 8.66 inches. For this range of data the amplification factor is still in an acceptable range of less than $2 \%$.

\section{BEAM TUBE DESIGN}

The HEB dipole design incorporates a novel beam tube assembly which provides for the dissipation of the anticipated $4.2 \mathrm{~K}$ heat loads during continuous ramping. The assembly allows for a redesigned flow loop that shortens the thermal path between the heat sink and the primary heat source. The new cooling scheme achieves more uniform coil temperatures through a continuous single phase to two phase heat exchange.

The beam tube assembly shown in Figure 4, consists of two concentric, constant thickness stainless steel tubes. The inner tube, 0.09375 inch thick, separates the bore tube vacuum from the two phase helium flow located in the annular space of the two tubes. This provides for a physical beam aperture of 1.6 inch vertical, and 2.2 inches horizontal. The two phase helium flows through an annulus 0.0625 inch wide at the mid-plane and 0.5 inch wide at the poles. The outer tube, 0.0625 inch thick, separates the single phase flow in the collared coil region from the two phase return flow. The single phase helium then flows through a 0.0625 inch gap concentric to the inside diameter of the coil. A thin $(0.003$ inch) copper plating is applied to the exterior of the single phase/two phase tube to moderate any azimuthal temperature distribution in the wall. Mechanically the inner beam tube is located with respect to the single phase/two phase tube by axially intermittent spacers. These spacers also integrate the tubes structurally by distributing the loads between the two. The single phase/two phase tube is located by contact with the collar packs in the pole region. This interference serves to limit the deflection of the tubes when pressurized and maintain material stresses within acceptable limits. 


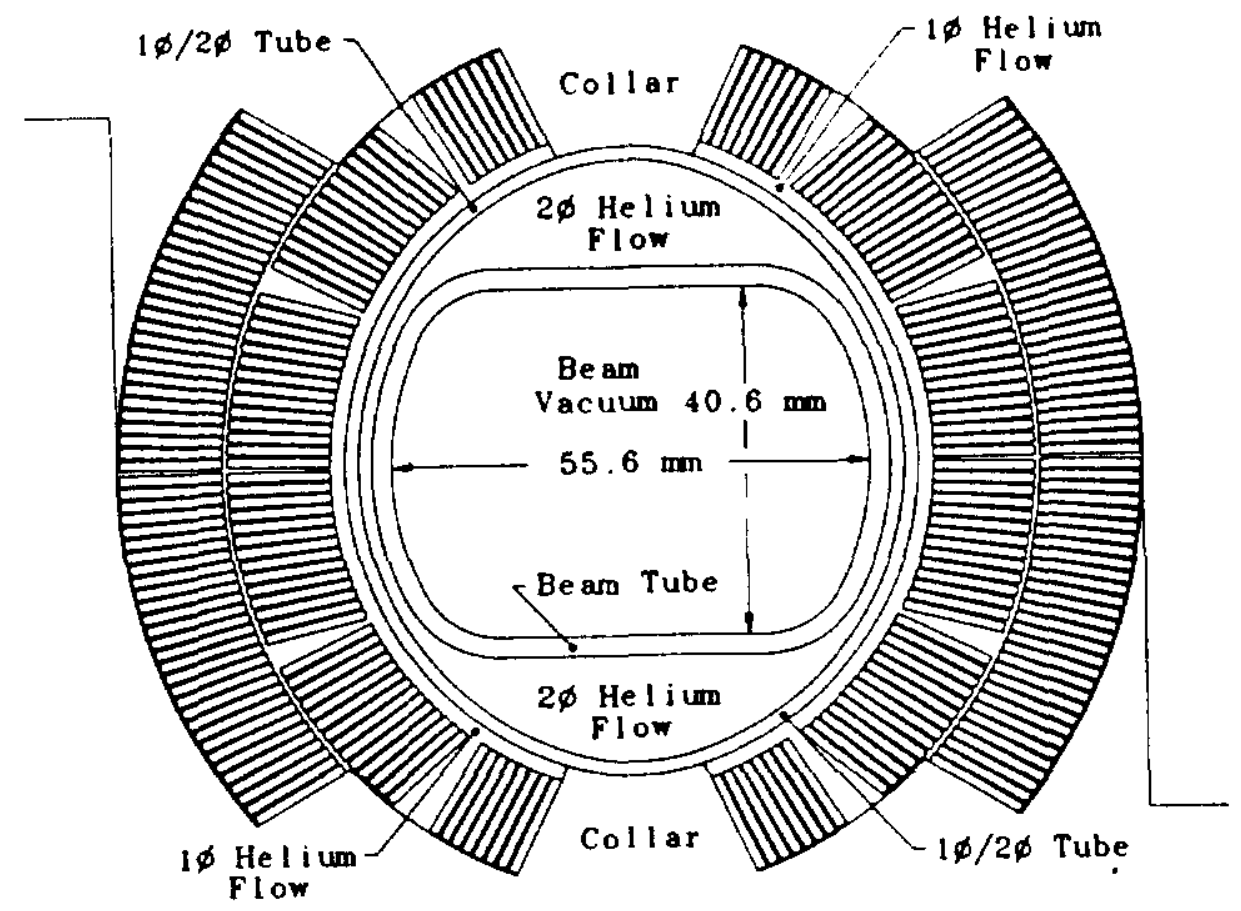

\section{COLLAR DESIGN}

Figure 4. HEB Dipole Beam Tube Assembly

The collar design of the HEB follows the collider ring magnet closely. The collars are high strength aluminium, 3.724 inches OD with front and back collars joined by semiperfs. The locking mechanism is provided by eight keys located symmetrically, two per quadrant. Unlike other designs the HEB dipole collars have a stainless steel skin. The

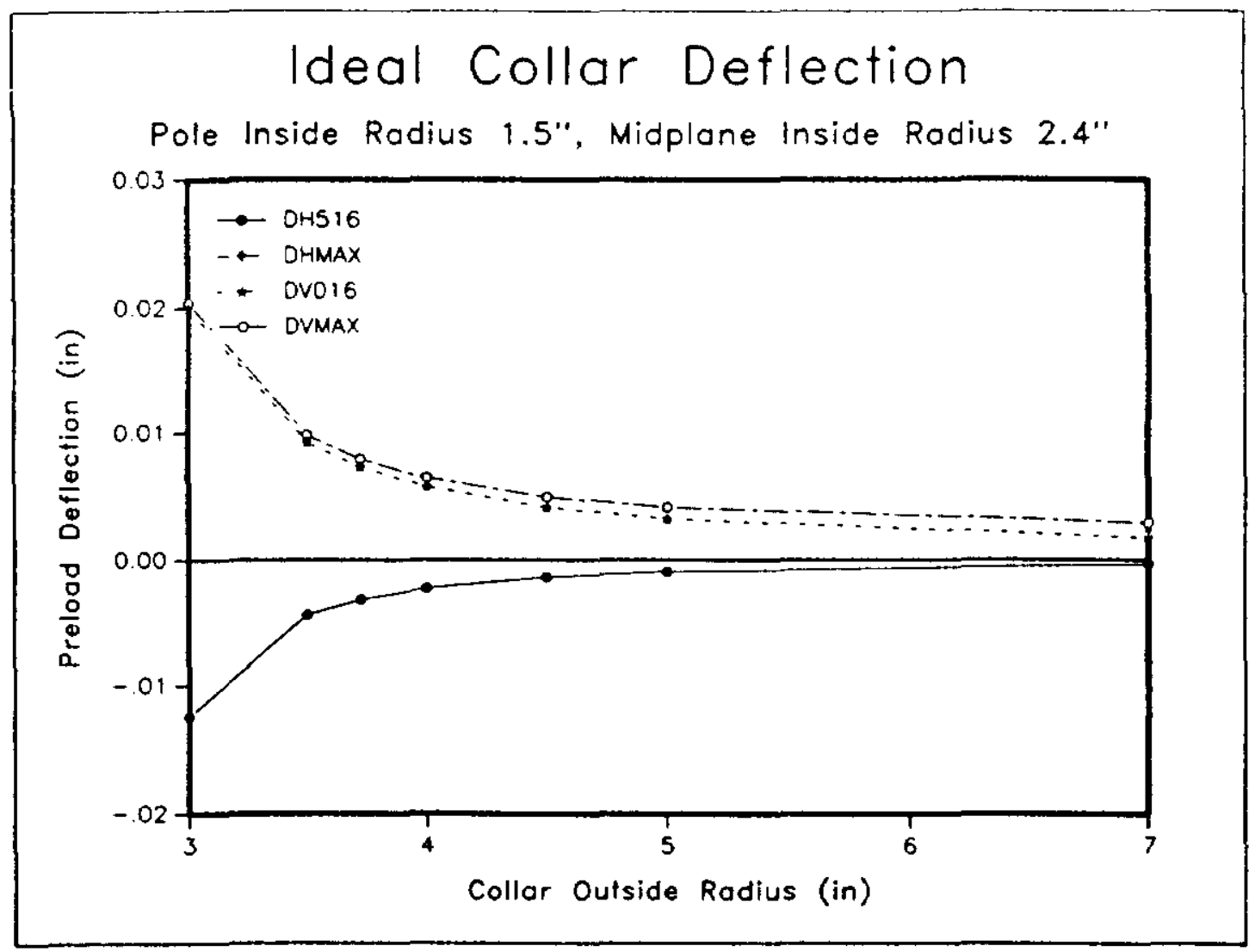

Figure 5. HEB Dipole Collar Deflection vs. Collar Thickness 
skin, 0.0625 inch thick, serves to limit the single phase helium volume, but is not a structural member in the assembly.

The magnetic analysis of the yoke saturation properties indicated that a yoke inner radius of 3.8 inches was optimum. This result coincides well with the structural analysis which indicates that a collar outer radius greater than 3.5 inches is needed. A collar outer radius of 3.724 inches was selected which results in 0.003 inch deflections in the horizontal parting plane and 0.008 inch in the vertical from preload forces without any yoke support.

With the collar dimensions chosen, a keying mechanism is needed which makes the actual collar approach the ideal case as closely as possible. The keys provide the locking mechanism which prevents the collars from springing apart when the collaring process is over. Furthermore, they must be sized so that the deflection of the collared coil is minimized while the maximum bending stresses remain below the elastic limit of the collar material.

Each of the collar keys are 0.2 inch wide each. The four keys nearest the horizontal midplane are 0.375 inch deep, the remaining ones are 0.25 inch deep. The keys are all stainless steel. The finite element model used for the analysis consists of front and back collars which, by symmetry, provide a complete model of the collar lamination packs. This method is identical to that used in the analysis of collider dipoles. Forces arising from $20 \mathrm{kpsi}$ inner and $10 \mathrm{kpsi}$ outer preloads (corresponding to a $8.8 \mathrm{~T}$ central field) were applied directly to the inner surface of the coil cavity in the collars. Keys were created of various size, shape, and number to simulate schemes of potential interest.

The first collar design used a single key per quadrant. The collar deflection was limited to 0.012 inch in the vertical plane. However a local maximum von Mises stress of $96 \mathrm{kpsi}$ was predicted in the collar at the midpoint of the keyway, 37\% larger than the elastic limit of the collar material. To distribute the stresses more evenly, a second key was added to each quadrant, both keys being 0.25 inch deep. The vertical deflection is reduced slightly, to 0.011 inch, and the maximum stress is reduced to $78 \mathrm{kpsi}$ at the midpoint of the upper key. The midplane key is shielded by the action of the upper key. Increasing the depth of the midplane key to 0.375 inch reduced the vertical deflection to 0.010 inch and reduced the maximum stress to $\mathbf{7 0} \mathrm{kspi}$ by distribuling the locking action of the keys more uniformly. This was the design chosen.

\section{YOKE MECHANICAL DESIGN}

The HEB dipole is a cold iron magnet with a vertically split, dry iron yoke. Cold iron has several advantages over its warm iron counterparts. First, it provides the maximum field enhancement due to the close proximity of the iron to the collared coil assembly. Second, there are no forces imposed on the suspension system resulting from the coil and iron centerlines not being coincident. The disadvantage of cold iron lies principally with the fact that it represents a significant load on the refrigeration system during cooldown.

There are two differences between the HEB dipole yoke and the collider dipole yoke. First, the HEB dipole uses a vertically split yoke. This ensures that a parting plane gap represents no perturbation to the magnetic field. Contact between the yoke and the collar is ensured in the horizontal plane. Mechanically this increases the cold mass resistance to the Lorentz forces by increasing the structural stiffness. This assembly style has recently been tested on collider dipole short magnet models and is also used in the sucessfully prototyped LHC dipole magnets.

Second is in use of dry iron. In this system the single phase helium flow is contained in two pipes 1.25 inches in diameter which pass through the yoke laminations. The advantages of the dry iron concept are that the total helium volume of the magnet is reduced and that the cold mass outer skin is no longer required to satisfy pressure vessel codes. This latter feature allows the yoke laminations to be cropped at the top and bottom thus reducing the yoke mass and providing space within the cryostat for the cryogenic 
piping. Concerns about the dry iron concept include ensuring thermal contact between the single phase pipe and the laminations at all times, and the quench characteristics of the magnet with reduced helium volume leading to significant pressure rises. The quench behavior is expected to be similar to the warm iron Tevatron dipoles where all the helium volume is contained in the collared coil region as in this magnet. Analysis of these effects is still in progress.

\section{COLD MASS SUSPENSION SYSTEM}

The suspension system in a superconducting magnet performs two functions; it resists structural loads imposed on the cold mass assembly during shipping, handling, seismic disturbances, and quenches, and it insulates the cold mass from heat radiated and conducted from the environment. The suspension system for the HEB dipole follows that of the collider dipoles. The major components of the suspension system are shown in Figure 6. The magnet assembly is supported vertically and laterally at two places along its length. To accommodate axial shrinkage during cooldown, the magnet assembly is free to slide on one support while the other serves as the anchor position. To distribute any imposed axial load to the supports, a tie bar is used to connect the supports. The structural design criteria are listed in Table 3.

Each post assembly consists of inner and outer composite tubes connected by an intermediate stainless steel transition tube. Stainless steel and aluminium discs and rings serve to join the tubes and act as tie points to other cryostat components. A cross section through a typical re-entrant support post is shown in Figure 7. Calculations and tests have shown that the bending loads resulting from the axial and lateral loads produce the highest stresses in the post assembly. In order to satisfy the load cases a computer program was written to calculate the tube thicknesses required to satisfy the structural requirements from a given set of input criteria. 4 The overall height and diameter of the support post and the ratios of the various thermal path lengths are determined in large part by the cryostat configuration and the conductive heat load constraints.

The support post design which satisfies the structural requirements results in a fiberglass composite outer tube 8 inches in diameter with a wall thickness of 0.14 inch. The inner tube is a 6 inch diameter graphite composite with a wall thickness of 0.15 inch. The maximum stresses are $20 \mathrm{kpsi}$ and $30 \mathrm{kpsi}$ respectively. The estimated heat load from this design to the $4.2 \mathrm{~K}$ system is $0.12 \mathrm{~W}$ per post assembly. The $20 \mathrm{~K}$ and $80 \mathrm{~K}$ heat loads are 0.82 and $6.2 \mathrm{~W}$ respectively.

\section{CRYOSTAT DESIGN}

The cryostat for the HEB dipole also has its origin in the SSC main ring dipole. The cryostat consists of a vacuum vessel, $80 \mathrm{~K}$ and $20 \mathrm{~K}$ shields, cold assembly, multilayer insulation (MLI), and suspension assembly. Unlike its collider counterpart, which will have a synchrotron radiation heat load to $4.2 \mathrm{~K}$ of $0.15 \mathrm{~W} / \mathrm{m}$, the $\mathrm{AC}$ heating due to ramping of the magnet to full field and back will result in a heat load of approximately $2.0 \mathrm{~W} / \mathrm{m}$. This higher heat load accounts for most of the differences in the piping schemes between the two cryostat systems particularly in the two phase cooling around the beam tube.

Table 3. HEB Dipole Suspension System Structural Design Criteria

Shipping and Handling Loads

Seismic load guidelines

Maximum axial quench load vertical $2.0 \mathrm{G}$

lateral $1.0 \mathrm{G}$

axial $1.5 \mathrm{G}$

Nuclear Regulatory Guide 1.61

1.61 vertical and horizontal spectra scaled by 0.3 $15000 \mathrm{lb}$ 


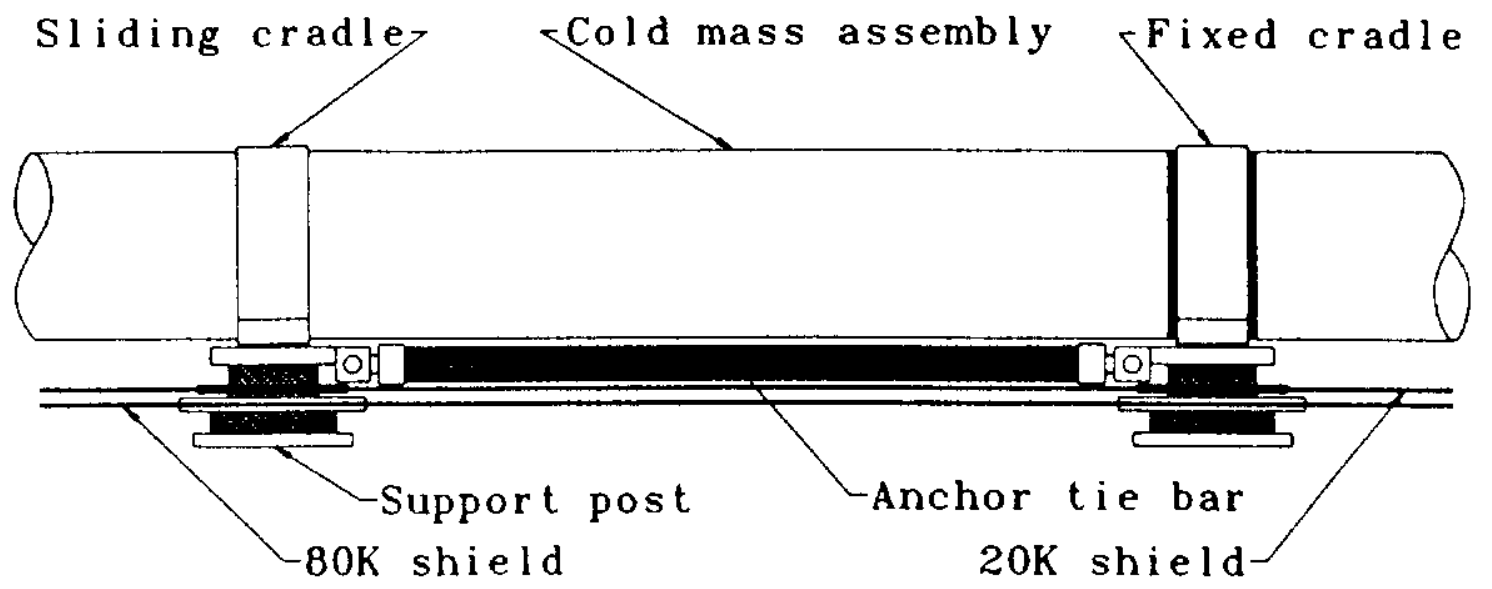

Figure 6. HEB Dipole Suspension System

The vacuum vessel is a 27 inch diameter carbon steel pipe. It is fabricated from three lengths of pipe joined at stiffening rings located at the two support post locations. The stiffening rings are required to transmit internally generated loads from the support posts to ground.

The two shells radially inward from the inner surface of the vacuum vessel are thermal radiation shields. They serve as heat sinks to minimize radiative heat transfer to the collared coil assembly. The $80 \mathrm{~K}$ shield intercepts heat radiating from the $300 \mathrm{~K}$ surface of the vacuum vessel. The $20 \mathrm{~K}$ shield intercepts heat radiating from the inner surface of the $80 \mathrm{~K}$ shield. Both shields also intercept heat conducted through the support system The shields are aluminum shells welded to their respective cooling tubes, and both shields are covered with MLI in order to minimize radiative heat transfer to their surfaces. The $80 \mathrm{~K}$ shield is covered with $3 / 4$ inch of reflective mylar and a nylon spacer material. Approximately $3 / 8$ inch cover the $20 \mathrm{~K}$ shield. A cross section of the complete cryostat system is shown in Figure 2.

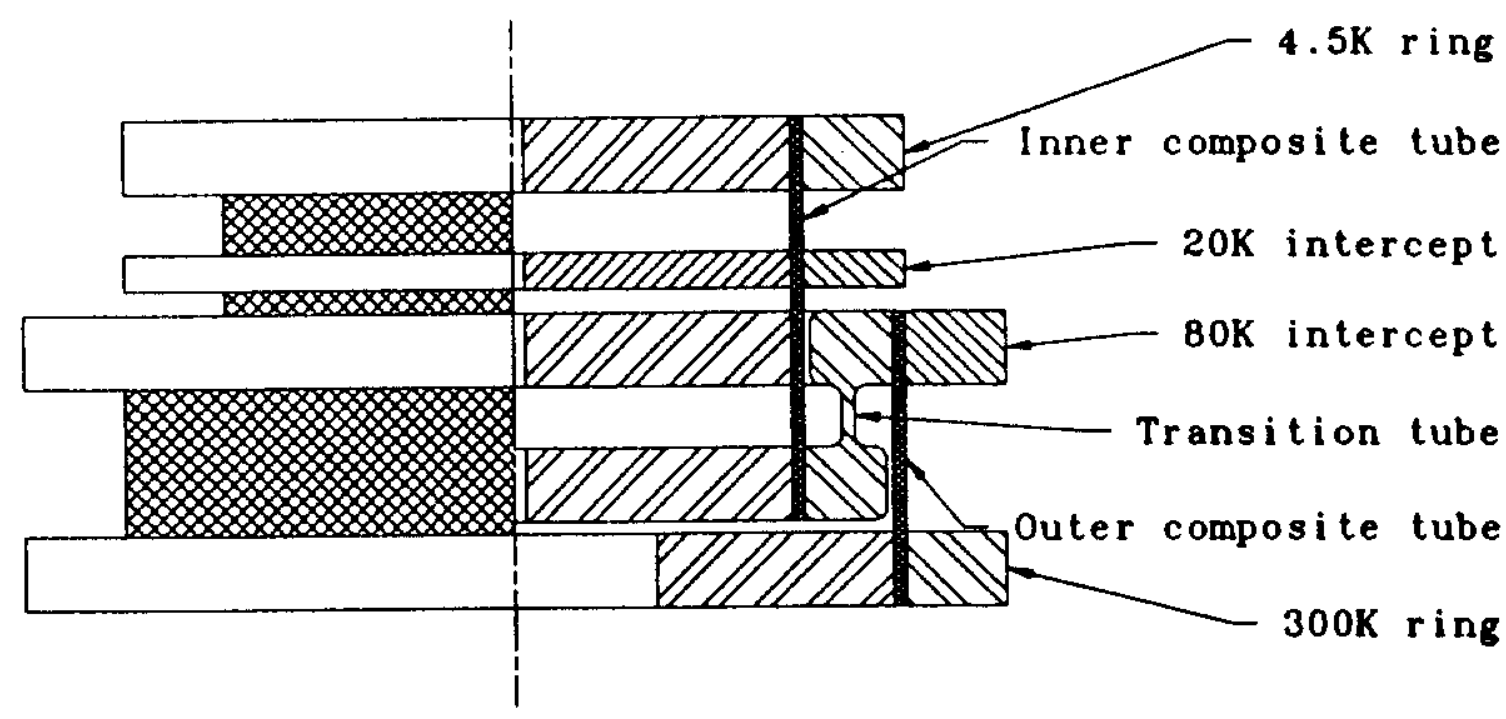

Figure 7. HEB Dipole Support Post Assembly 
As mentioned in the introduction, a complete description of the design work to date on the high energy booster is beyond the scope of this paper. We have tried to present an accounting of the areas in which we have made significant progress and which represent interesting aspects of the design. This work is clearly not the last word in the HEB design. Much R\&D needs to be done, particularly in those areas of the magnet which represent new or unproven technology, e.g. the offset coil geometry, the annular two phase flow inside the beam pipe, indirect iron cooling, etc. Also the details of the lattice need to be addressed so that the physical parameters of the dipole can be finalized. However, we feel that this work serves as a good starting point from which a final design can begin.

\section{REFERENCES}

1. SSC Laboratory, "Site-Specific Conceptual Design Report," November 22, 1989.

2. T.Collins, Fermilab Internal Report, TN1406.

3. A.Ishibashi, et al., Design study on the superconducting dipole magnets with noncircular aperture coils in application to future colliders, in: "Supercollider 1," Volume 1, Plenum Press, New York.

4. T.H. Nicol, et al, Design and analysis of the SSC dipole magnet suspension system, in: "Supercollider 1," Volume 1, Plenum Press, New York. 